\title{
POLAND's Path TOWARdS Decompression REalisation IN UNDERWATER WORKS Part II. DECOMPRESSION TABLES FOR COMMERCIAL DEEP-SEA DIVING IN POLAND
}

\author{
Stanisław Skrzyński \\ Faculty of Mechanical and Electrical Faculty, Polish Naval Academy in Gdynia, Poland
}

\section{ABSTRACT}

In the article the author presents the specificity of decompression of deep-sea dives in relation to methods used in underwater works, with particular emphasis on commercial diving in our country. In the 50 - $90 \mathrm{~m}$ depth zone in the Polish offshore area, decompression was used in underwater works (works below the depth of $50 \mathrm{~m}$ ) based on tables designed for bell diving. The technical, organisational and formal conditions of these underwater works corresponded to the national diving potential. The implementation of decompression, particularly in deep-sea dives, provides the possibility of performing it in many different ways. They, in turn, are the result of experience, diving technique, organisation and specificity of underwater works.

The article presents the implementation of decompression from the executive side of underwater deep-sea works developed and carried out by the Department of Underwater Works Technology of the Naval Academy in cooperation with the Navy until 2001 and civil companies to date.

Keywords: diving computer, application of diving computer diver's decompression, deep underwater work, deep diving, professional diving, data deep diving, diving bell, breathing mixtures, decompression table.

\section{ARTICLE INFO}

PolHypRes 2019 Vol. 69 Issue 4 pp. $45-62$

ISSN: $1734-7009$ elSSN: $2084-0535$

DOI: $10.2478 / \mathrm{phr}-2019-0019$

Pages: 18, figures: 0 , tables: 2

page www of the periodical: www.phr.net.pl

\section{Review article}

Submission date: 22.02.2019 r. Acceptance for print: 23.04.2019 r.

Publisher

Polish Hyperbaric Medicine and Technology Society 


\section{INTRODUCTION}

In the first part of the article, the author presented the current state of deep-sea diving in a world in which it can be seen that deep-sea diving for commercial purposes has, for the last two decades, been subject to stagnation. This trend resulted in a lack of change in attitude towards deep-sea diving, a fact that is reflected in the normative documents in relation to diving for other purposes, in particular military.

In our country the base for deep-sea diving is not developing. Even the implementation in the Navy of the diving system purchased in 2018 using a wet bell has not improved this situation. This system has only increased deep-sea diving capabilities. The wet bell does not increase the efficiency of deep dives; when no surface decompression is used, the whole dive, even the intermediate and shallow oxygen decompression stops, takes place in water. World diving standards recommend the use of a wet-type diving bell to a depth of 75-80 m, but with very short diving times of up to $30 \mathrm{~min}$ including the process of descent $[1,2,3]$. The effectiveness of the wet bell can be increased by using water-heated suits (which I do not recommended) or electrically heated suits.

\section{DEEP-SEA DIVING IN OUR COUNTRY UNTIL}

\section{8}

Deep-sea submarine works for the offshore industry in Poland (performed by a company co-owned by the USSR, the GDR and the People's Republic of Poland) started in 1982 and were carried out intermittently until 1987. The works were carried out by the Naval Rescue Ship ORP "Lech", based on equipment from the German company Dräger. The diving technology developed in the Naval Rescue Headquarters of the Polish Navy was based on US Navy heliox tables, adopted for the umbilical semiclosed circuit devices FGG-III with constant mixture dosage. The duration of the divers' stay in depths of 70-80 $\mathrm{m}$ was up to $30 \mathrm{~min}$ and the complete dive cycle from 4 to 4.5 hours. A pair of divers started the dive in the apparatuses while staying in the bell, the helix decompression process took place in the bell, and oxygen decompression from $12 \mathrm{~m}$ in the chamber.

During decompression with a mixture containing $60 \%$ oxygen, the divers inhaled using the adopted KP-18 airborne inhalers and exhaled into an installation supplied by the Germans in which an injector device removed the exhaled mixture outside the chamber. During these dives there were several cases of a light form of decompression sickness, in the author's opinion caused by a slight difference in the selection of decompression for the semiclosed circuit devices in relation to the injector equipment for which American tables were used.

Due to the economic situation and political changes in Poland, underwater works for the benefit of the Polish offshore industry were reinstated in 1993. They were carried out with the same FGG-III devices adapted to the new technology, oxygen inhalers produced in Poland, and other techniques produced by domestic manufacturers. This technique corresponded to the applied diving technology, developed and tested in the Department of Diving Equipment and Underwater Works Technology of the Naval Academy in cooperation with the Department of Maritime Medicine of the Military Medical
Academy (WAM). The technology, based on trimix decompression tables modelled on the French Dorris tables, used for decompression, in addition to a working mixture appropriate for a given depth range, air and oxygen at stops from 12 to 3 metres. In order to cover the 50-120 m depth zone it was necessary to use four trimix mixtures with an oxygen content of $20 \%, 16 \%, 13 \%$ and $10 \%$. The organisation of the dive envisaged a diving bell and return with decompression performed in the bell and then in the chamber. During oxygen decompression, 5 -minute air breathing breaks were used after every 30 minutes of breathing oxygen to reduce the risk of oxygen poisoning. FGG-III devices were used until 1996.

Since 1997, based on the above mentioned trimix diving technology, open-circuit helmets and full masks were adopted using French company COMEX and helmets of the American company Superlite. Until 2007, deep-sea diving technology based on Polish trimix tables was carried out from the dive bases of the rescue vessels ORP "Lech" and ORP "Piast", mobile diving systems Af-2, and the MOBNUR deep-sea diving system developed by the Department of Underwater Works Technology of the AMW. These systems were installed on oil platforms.

This technology was used for underwater commercial works in our country until the introduction of the official tables contained in the Regulation of the Minister of Health dated 17 September 2007 on health conditions for the performance of underwater works on the basis of art. 11 (6) of the Act on the performance of underwater works [4]. The regulation made the performance of underwater works more difficult by imposing predesigned decompression tables and linked them to specific diving techniques and medical security. Unfortunately, thereby, the chapter concerning the application of Polish experimental solutions and deep-sea diving methods, based on Polish intellectual and technical potential, was closed.

\section{IMPLEMENTATION OF STATE (STATUTORY), OFFICIAL DECOMPRESSION TABLES FOR DEEP-SEA DIVING}

Since 2008, the helix tables provided in the Ordinance of the Minister of Health on 17 September 2007 have been obligatorily used [5] within the deep diving zone of $50-120 \mathrm{~m}$. The above-mentioned regulation contains three types of tables, used depending on the diving technique and the composition of the working and decompression mixture and oxygen used in the last decompression phase. Below I present a brief description of these tables.

I -heliox/oxygen/6 m tables - [app. 5 it. 2] in the interpretation of the application of these tables, "Decompression and compression procedures for a heliox with oxygen decompression at a depth of 6 meters" recommend the following:

- The tables are designed for diving at depths between $30 \mathrm{~m}$ and $60 \mathrm{~m}$. They contain the socalled abort tables, i.e. dive interruption tables allowing to interrupt the dive above the depth of $30 \mathrm{~m}$.

- The break following the dive is at least 12 hours. During this break no subsequent dive is 
permitted, irrespective of the gas used for breathing.

- Diving can be performed wearing independent diving equipment (this is not in accordance with the Regulation of the Minister of Infrastructure of 19 May 2004 on safety and hygiene at work in the performance of underwater works, not limited to underwater deep-sea works [13]), with umbilicalled apparatus or in a diving bell with restrictions resulting from the selected method. Implicitly, diving with the use of a platform, a tether rope, and, of course, a diving bell.

\section{DECOMPRESSION PROCEDURES}

1. the speed of ascent to the first stop should be 9 $\mathrm{m} / \mathrm{min}$ and must not exceed $15 \mathrm{~m} / \mathrm{min}$,

2. decompression in water with stops every $3 \mathrm{~m}$ to a depth of $6 \mathrm{~m}$,

3. the last minute of the decompression stop time is used to ascend to the depth of the next stop,in the final decompression phase, during the last minute, the diver emerges from the $6 \mathrm{~m}$ stop directly to the surface [5].

The above-mentioned tables cover the depth zone from 51 to $60 \mathrm{~m}$ using heliox mixtures $18-20 \% \mathrm{O}_{2}, 20-22 \%$ $\mathrm{O}_{2}$, and $22-24 \% \mathrm{O}_{2}$.

The decompression options are given every 10 minutes at depths of 30-68 m, and the maximum stay times for the deep zone are from 90 to 50 minutes for the zone's boundary depths. These tables use oxygen for decompression from a depth of $6 \mathrm{~m}$ onwards. The intention of such oxygen decompression is its implementation in the water, which is allowed in several countries of the world. Our national normative acts prohibit oxygen decompression and require the use of a diving bell, however, they do not indicate the preferred type of a diving bell. The tables are applicable for each type of a diving bell.

II - heliox/oxygen/12 m tables - [app. 6 it. 2] in the interpretation of the application of these tables, "Decompression and compression procedures for a heliox with oxygen decompression at a depth of 12 meters" recommend the following:

- The use of decompression tables covers a depth range from 30 to $78 \mathrm{~m}$. The tables include dive abort tables to interrupt the dive above the depth of $30 \mathrm{~m}$.

- The minimum dive interval after using the heliox/oxygen/12 m tables is 12 hours. During this time, repeated dives are not allowed regardless of the breathing mixture used.

- Wet bell diving method only. During decompression two divers are required to be present in the bell and secured against bell flooding. At the time of introduction of these tables no operational wet bells were in use in our country. When implementing these tables for underwater works, the Af- 2 and MOBNUR dry type open bells were used. Flooding of these bells was foreseen as the last resort, only in an emergency situation, in order to facilitate the manoeuvring of an injured diver in to the bell.

\section{DECOMPRESSION PROCEDURĘ: (IDENTICAL AS IN THE CASE HELIOX/OXGEN/6 M TABLES)}

\section{BREATHING MIXES}

The mixture used at the bottom is a heliox mixture with an oxygen content for which P02 is between $850 \mathrm{hPa}$ (0.850 bar) and $1550 \mathrm{hPa}$ (1.550 bar).

\section{DECOMPRESSION MIXES}

- the ascent to the first decompression stop is performed using the mixture used at the bottom, regardless of the depth of the first stop,

- for stops deeper than $30 \mathrm{~m}$, the diver breathes the working mixture used at the bottom,

- for stops between $30 \mathrm{~m}$ and $12 \mathrm{~m}$, the diver breathes air, or a mixture used at the bottom with a percentage of oxygen above $21 \%$,

- for stops between $12 \mathrm{~m}$ and the surface the diver breathes through the mask for 25 minutes with oxygen and then 5 minutes with air from the bell. If the bell was filled with the mixture used at the bottom, it should be replaced with air for stops starting from a depth of $12 \mathrm{~m}$.

The above interpretation is contained in the Regulation. However, it omits the fact that the tables can be used for any type of diving bell, like all the tables in the regulation. The advantage is that the choice of decompression method is based on the depth times given every 10 minutes. These tables use air for decompression, while $20-22 \% \mathrm{O}_{2}$ heliox is used to a limited extent. For a $17-18 \%$ heliox mixture, covering a depth zone of $50 \mathrm{~m}-$ $78 \mathrm{~m}$, air is the primary decompression mixture and oxygen is used from the depths of $12 \mathrm{~m}$ to $6 \mathrm{~m}$. The same is true for the 50-69 m zone using the 18-20\% heliox working mixture. In the 51-60 m zone, on the other hand, $20-22 \%$ heliox is used, which optionally also serves as a decompression mixture as an alternative for air.

The operational stay times in the tables for extreme depths of $50 \mathrm{~m}$ are $90-100 \mathrm{~min}$ and for depths of $75 \mathrm{~m}$ the maximum stay time is $50 \mathrm{~min}$. The $78 \mathrm{~m}$ table is used as a back-up and emergency decompression method.

III -heliox/bell tables - [app. 7 it. 2] used in closed and open bell dives and equipment enabling TUP operations to transfer pressurised divers from the bell to the hyperbaric chamber. For decompression options, the depth time is given every 15 minutes. Minimum and maximum stays are fixed for the entire 50-120 m depth zone from 15 min to 2 hours. The tables include dive abort tables, allowing to interrupt the dive above $30 \mathrm{~m}$ using the decompression mixture.

The above-mentioned depth zones are covered by overlapping ranges of the same oxygen partial pressure levels, corresponding to six mixtures, including the decompression mixture, as shown in Table 1. 
Depth zones for heliox mixtures [5]

\begin{tabular}{llll}
\hline No. & $\begin{array}{l}\text { HELIOX WORKING } \\
\text { MIXTURE }\end{array}$ & $\begin{array}{l}\text { DEPTH } \\
\text { UNDERWATER WORKS }\end{array}$ \\
\hline 1. & $20-22 \%$ Heliox & $50-60 \mathrm{~m}$ & \\
\hline 2. & $18-20 \%$ Heliox & $50-69 \mathrm{~m}$ & \\
\hline 3. & $16-18 \%$ Heliox & $50-78 \mathrm{~m}$ & \\
\hline 4. & $14-16 \%$ Heliox & $50-87 \mathrm{~m}$ & \\
\hline 5. & $12-14 \%$ Heliox & $60-99 \mathrm{~m}$ & \\
\hline 6. & $10-12 \%$ Heliox & $72-120 \mathrm{~m}$ & \\
\hline
\end{tabular}

This offers many diving options for the same depths, which implies different decompression times. Table 2 shows examples of decompression times, depending on the mixture used for the depth of the Polish shelf and the most frequently selected times for decompression choice.

Decompression times for typical depths of works on the Polish shelf, depending on the mixtures used.

\begin{tabular}{llllll} 
Depth [m] & $\begin{array}{l}\text { Stay time } \\
{[\mathrm{min}]}\end{array}$ & $\begin{array}{l}16-18 \% \text { Heliox } \\
{[\mathrm{h} / \mathrm{min}]}\end{array}$ & $\begin{array}{l}14-16 \% \text { Heliox } \\
{[\mathrm{h} / \mathrm{min}]}\end{array}$ & $\begin{array}{l}12-14 \% \text { Heliox } \\
{[\mathrm{h} / \mathrm{min}]}\end{array}$ & $\begin{array}{l}10-12 \% \text { Heliox } \\
{[\mathrm{h} / \mathrm{min}]}\end{array}$ \\
\hline 70 & 45 & $3: 59$ & $4: 54$ & $5: 16$ & $5: 33$ \\
\hline 80 & 45 & - & $6: 09$ & $6: 39$ & $6: 24$ \\
\hline 87 & 45 & - & $7: 09$ & $7: 32$ & $7: 49$ \\
\hline 70 & 60 & $5: 41$ & $6: 55$ & $9: 01$ & $7: 42$ \\
\hline 80 & 60 & - & $8: 34$ & $10: 09$ & $9 ; 35$ \\
\hline 87 & 60 & - & $9: 41$ & & $10: 36$ \\
\hline
\end{tabular}

For decompression, $20-22 \% 02$ heliox is used for all heliox mixtures regardless of the dive depth, and oxygen from a depth of $12 \mathrm{~m}$.

The introduction of domestic decompression tables did not take into account all aspects of the commercial diving environment in our country. For years, the national technical base for deep-sea diving was being prepared for the use of trimix mixes, while the state tables introduced heliox mixes. In the author's opinion, this was a result of interference from the medical civil environment, without experience in the field of deep-sea diving, since for over a quarter of a century, it was the responsibility of military doctors to secure these dives. Moreover, the tables, despite their many advantages in application, do not cover the whole deep-sea diving system as was the case with the trimix diving system developed in the Department of Diving Appliances and Underwater Work Technology of the Naval Academy (current name: Department of Underwater Works Technology) with significant participation in the form of the development of tables and medical protection of the Department of Marine Medicine of the Military Medical Academy. What is most important, when using open-circuit breathing equipment, these were tables that reduced the helium consumption, thus generating lower costs and improving the diver's thermal comfort.

From the point of view of decompression from deep-sea dives, the decompression table system should include emergency tables in the form of abort tables, mixture and decompression gas exchanges, and violation of acceptable diving parameters, including the system of medical recompression tables and emergency saturated dives. The introduced tables absolutely require official supplementation with the above-mentioned factors and procedures. When implementing the official tables, we based our work and experience on the work and experience of the Department of Underwater Works Technology of the Medical University of Warsaw and diving doctors of the Department of Maritime Medicine of the Military Medical Academy. The consultations with the authors of the regulation, for obvious reasons, i.e. lack of experience in conducting this type of dives, did not help to solve many specific aspects of technologies based on these tables. The best advice the author received from them was to "follow the interpretation of the tables", something that was done throughout the implementation anyway. The problems in particular resulted from the specificity and possibilities of the diving technique.

The decompression tables (French COMEX 1992 and French Navy - Marine Nationale 90 (MN90) decompression tables), which were obligatory for commercial deep-sea diving, corresponded to the French diving technique and diving facilities. This excluded the possibility of using other decompression tables for the already existing technique and technology. The regulation in its requirements also limited the group of doctors authorised to secure medical works at depth. Physicians with specialisations that do not include elements concerning hyperbaric and diving (anaesthesiology) in their programme were indicated, whereas specialisations dedicated to pressure changes (marine medicine, transport medicine or aviation medicine) were omitted $[4,5]$.

Any underwater work carried out deeper than 50 $\mathrm{m}$ is a compromise between the task being carried out and 
the tables in force, which impose the method of its execution. The safety requirements of underwater works, despite their low efficiency, require precise preparation of the execution variants and organisation of work, due to the limitations of the diver's stay at a depth of less than 120 min, including the descent and the bell lifting operation. The tables introduced by the above-mentioned Regulation provide great opportunities to use many diving options for a wide range of safety techniques and for underwater works, but like all the tables for deep diving, they require specific working mixtures in particular depth zones. Unfortunately, the technical condition of the national diving base does not give the full possibility to use them [7] due to technical reasons and very poor demand for deepsea underwater works.

Adaptation to heliox mixtures and making use of the advantages of the new decompression tables contained in the above mentioned regulation would require replacement of the diving technique developed in our country. Therefore, the diving technologies have been adapted to their use without major changes to the only operational diving technique available in our country, i.e. the mobile Af- 2 system, designed for saturated dives and modernised twice in our country to ensure international safety standards. The use of this system allowed deep-sea dives to exploit all the assets resulting from the use of saturated dives, i.e. the comfort of remaining in a heated diving bell, a full monitoring of the divers via the underwater TV (through the bell's camera system), monitoring of the atmosphere composition of the diving bell and hyperbaric chamber, as well as the possibility of staying in the hyperbaric chamber, which provides a significant level of decompression comfort. It was decided not to use heated suits, assuming the average duration of the diver's stay at a depth of 40-55 min and, if necessary, up to 60-75 min. For these average staytimes and medium heavy workloads, an undersuit and a dry suit constitute sufficient heat protection for the diver.

While starting to apply the official tables, the author sought an answer to the question how these tables would hold up under the conditions where the divers were working hard and whether the so-called "good diving practice" in this case would yield a positive result. French tables were verified on a group of standard anthropometric data of French Navy divers in 1988 with the following data: weight $74 \mathrm{~kg} \pm 8 \mathrm{~kg}$, height $175.9 \mathrm{~cm} \pm$ $5.7 \mathrm{~cm}$, age 32.3 years \pm 6.1 [3].

There have not been many deep-sea divers with these anthropometric data trained in our country for over 30 years. If we add to this situation very heavy or heavy underwater work performed by divers, such as assembly work performed with heavy wrenches, hydraulic tools, working with ejector, cleaning the surface of underwater structures, etc., the author believes there was a high probability of an occurrence of decompression incidents. These conditions include possible accidents at work, resulting from the profile of underwater work, which is classified as high-risk work, the effects of which could disrupt the decompression process.

All of the above factors required extended decompression, the parameters of which could not always be provided by the tables when using particular heliox mixtures. In such cases, "undecompressed" diving factors, i.e. the adopted organisation of underwater work and diving technique, came to the aid of the diving technology.

The implementation of domestic heliox tables [5] was carried out after prior analysis and preparation of the technology, taking into account the national technical base at the Department of Underwater Works Technology. In this case, the dive base and diving technique were adjusted to the decompression tables. Underwater works were preceded by the preparation and training of diving crews, and the examination of several variants of the diving course in port and at sea, where the Af- 2 diving system was used.

The technical and operational parameters of the diving bell, supply installation, and the lifting equipment of the Af-2 diving system were conducive to "improving" the decompression, i.e. reducing the inert gas saturation of tissues in the diver's body. In the period from September 2008 to February 2018, 446 dives in the depth zone of 50$87 \mathrm{~m}$ were performed for underwater works purposes on the Polish shelf without any decompression incident, for stays of 45 to 60 minutes. In addition, dives with stays of up to $90 \mathrm{~min}$. and decompression lasting more than 13 hours were performed. This state of affairs, in the opinion of the author, apart from ensuring high comfort of decompression, was influenced by factors that are analysed in the next chapter.

\section{IMPLEMENTATION OF DECOMPRESSION IN UNDERWATER DEEP-SEA WORKS WITH THE USE} OF DOMESTIC TECHNIQUE

During immersion in an open bell, operations are carried out to keep the inside of the bell 'dry' and this requires a specific controlled supply of the bell (air or mixtures). Such a solution slowed down the process of immersion to $8-10 \mathrm{~m} / \mathrm{min}$. This speed is about $1 / 3$ of the table-admissible speed of $30 \mathrm{~m} / \mathrm{min}$. The time of the dive is also added to the time of the control dive for the divers.

Since the time of the diver's stay at depth, which is the basis for the selection of decompression, is included in the dive time, it results in a shorter real time of the diver's stay at the depth, which should be matched by shorter decompression. The working mixture that the diver breathes at depth is a hypoxic mixture containing less than $16 \%$ oxygen, only to a depth of $20-25 \mathrm{~m}$ does he breathe a mixture containing $20-22 \%$ oxygen, or a similar composition used during decompression.

The second factor improving decompression or increasing the conservatism of the tables (reducing the degree of saturation of the diver's tissues) are technical and organisational conditions.

The diving bell is never set at the depth of the diver's work, i.e. the pressure inside is lower. The differences in these depths for works on the Polish shelf range from a minimum difference of $3 \mathrm{~m}$, resulting from the ballast position of the bell's guide ropes (1.5-2 m from the bell's hatch and at least $1 \mathrm{~m}$ above the bottom or object), to $12 \mathrm{~m}$ when working on underwater mining structures (the bell and the ballast are placed above the extraction structure, whose height can reach from 7 to $9 \mathrm{~m}$ and sometimes more). A diver working at depth returns to the bell as a rule about 2 to 5 minutes before the time of lifting of the bell.

This time is also included in the stay time base for decompression selection. Being at the level of the bell, the diver prepares to ascend and enter the bell by laying his umbilical-hose bundle (reaching a minimum of $30 \mathrm{~m}$ and more), cleaning the suit and unloading or attaching tools. From the point of view of the decompression theory, the time of preparation for lifting the bell, depending on the 
depth and working time of the diver, until the moment of entering the bell can be treated as an additional decompression stop (deep stop) or as a two-level dive, lowering the resultant working depth of the diver.

The third factor concerns only the "heliox/bell tables", most commonly used in underwater deep-sea operations. In this case, the factor improving the efficiency of decompression in dives is increasing the oxygen content of the mixture in the bell space during ascent, until the divers switch to breathing with the decompression mixture. The principle has been adopted to increase the oxygen content in the diving bell atmosphere so that the oxygen content at the stop when the decompression mixture is introduced reaches the same value as in the decompression mixture included in these tables, i.e. at least $20 \%$. For example, a typical $78 \mathrm{~m}$ deep working mixture contains $14-16 \%$ oxygen.

The atmosphere of the bell at the beginning of its immersion contains about $21 \%$ oxygen from the air. In the initial phase of immersion, the bell is supplied to a depth of 5-8 m with a decompression mixture containing 20-22\% oxygen, and then with the working mixture, so that the bell operator and the working diver outside the bell breathe the same mixture. During the immersion, the diver's hose is 'flushed' with the working mixture, which results in the oxygen content of the bell's atmosphere during the immersion, and to a depth of approximately half the working depth, being greater than the content in the working mixture.

The authors of the tables claimed that in order to maintain the values indicated in the tables, the bell space must be ventilated so that the operator breathes the same mixture as the diver. After ventilating the bell with a working mixture containing $15 \%$ oxygen for the $3-4$ changes in its atmosphere, we arrive with a mixture containing $15.5 \%$ oxygen and $19 \%$ nitrogen, including the ventilation of the bell at sea level. The Af- 2 system in this case requires about 12-14 m3 of expensive heliox mixture. If the ventilation is carried out at depth, the amount of gas for ventilation at a depth of $0 \mathrm{~m}$ should be multiplied by the absolute pressure at that depth. This is an extremely expensive and time-consuming operation, which in a strict time regime for the implementation of deep diving cannot be afforded for safety and logistical reasons.

FACTORS DETERMINING THE IMPLEMENTATION OF DEEPSEA DECOMPRESSION WITHIN THE CURRENT NATIONAL TECHNICAL BASE

The basic factors that we have to analyse and take into account when performing decompression are:

- the moment when the working mixture of the bell atmosphere is replaced with the decompression mixture or when the working mixture is left,

- filling the chamber with a decompression mixture or optionally with air, and choosing the pressure of the decompression stop to collect the divers from the bell,

- what kind of dive organisation to adopt out of two options: the first variant being a working diver and a bell operator, the second being two divers in the water.

The transition from breathing with the working (bottom) mixture to the decompression mixture in accordance with the tables in force in Poland in the 50-100 $\mathrm{m}$ zone takes place at stops of $24 \mathrm{~m}$ or $30 \mathrm{~m}$ (shallower for the $18-20 \%$ oxygen heliox, greater for $20-22 \%$ oxygen, for the table depth of $51 \mathrm{~m}$ ) up to $45 \mathrm{~m}$ for the table depth of $102 \mathrm{~m}$. At stops from $12 \mathrm{~m}$ and more, oxygen in the decompression heliox atmosphere is used.

The TUP-transfer operation under pressure to move divers to the chamber to continue decompression can take place at any time during decompression. The last stop where the TUP-transfer operation should be performed is $15 \mathrm{~m}$, although in practice it is performed from deeper stops to give the divers time to undress, use the toilet, enable a light meal, "hydrate the body" and prepare for oxygen decompression. The following factors determine the choice of depth of the stop, from which the bell is lifted after sealing the hatch:

- heliox mixture savings, in the 50-75 m depth zone (air is used for decompression in addition to the working mixture, only with the adaptation of heliox/12 $\mathrm{m}$ tables),

- the time of the decompression stop where it is possible to perform the transfer to the chamber,

- the deterioration of the sea surface conditions that impede the TUP operation,

- an emergency situation forcing the evacuation of divers to the surface,

- loss of communication or gas supply (the bell has communication and emergency power channels), Only two of the above factors, which can be planned, result from improvements in efficiency and the economic and logistical rationale for underwater works.

\section{VARIANT I OF DECOPRESSION REALISATION}

Preparation for the decompression phase is already underway at the point immersion. The oxygen content in the bell atmosphere during the submerge cycle is increased by a few percent (2-3\%), which does not significantly change the oxygen partial pressure due to the fact that the bell stands at a shallower depth than the working depth of the diver. In no case should a one-time dose of oxygen toxicity provided for in the tables be exceeded. The tables are prepared for mixtures whose composition may vary by $2 \%$ in extreme cases.

For example, from a depth of $85 \mathrm{~m}$ for a $14-16 \%$ heliox, the same decompression is foreseen for a $14 \%$ mixture and for a $16 \%$ mixture and their intermediate values. The maximum oxygen partial pressure deviation for the case under consideration can be 0.19 ata $(19 \mathrm{kPa})$, which, for example, for a working mixture of $14-16 \%$ oxygen corresponds to a depth difference of about $12 \mathrm{~m}$. The oxygen partial pressure for $16 \%$ content at $85 \mathrm{~m}$ depth corresponds to a depth of $87.5 \mathrm{~m}$ for a content of $14 \%$.

In this case there is no possibility to exceed the dose of oxygen toxicity, which is ensured by the construction of tables, calculated for $2 \%$ changes in typical mixtures used in them. This is the range within which we move when increasing the oxygen content in the bell atmosphere until the oxygen content reaches $20 \%$. The same is true of the decompression mixture for the heliox/bell tables, where the oxygen content can be used in the range of $20-22 \%$.

During decompression, when oxygen is added to the working mixture to be used for breathing, increasing its content to $20 \%$, when the diver has to switch to breathing with the decompression mixture, it can be stated with certainty that even a large deviation (of the order of $3 \%$ ) from this content does not reduce the safety of decompression, as larger oxygen windows at the beginning of decompression than those provided for in the tables 
compensate for lower oxygen content at depths. As a rule, the decompression is controlled so as to achieve the required oxygen content.

Such decompression method is possible only in the bell prepared for saturated dives, i.e. the Af- 2 diving system bell. The bell allows ongoing monitoring and correction of oxygen content. This decompression method is only possible:

- when using "heliox/bell" tables,

- organisation of diving in the combination working divers-bell operator, i.e. one diver working in the depths and the bell operator securing him,

- the possibility of continuous measurement of partial pressure or oxygen content,

- $\quad$ fitting the bell with a dosing or controlled oxygen addition system.

In our practice we use a dosing system, which needs time. A better solution is a system of oxygen addition, which allows to increase the oxygen content of the bell in a short time by 3-5 \% for a given depth. The formation of oxygen pockets in the bell is unlikely due to the continuous circulation caused by the operation of fans of the absorber and heater. Despite the author's protests, the diving managers vented the bell, thus wasting the heliox decompression mixture to rapidly increase the oxygen content (which at a depth of $36 \mathrm{~m}$ would require about $45 \mathrm{~m} 3$ of the decompression mixture to increase the oxygen content by about $2 \%$ ). Ventilation causes noise and interferes with the oxygen sensors (especially in winter conditions, when a cold gas has contact with the sensor and generates thermal "shock" errors) without any significant effect on oxygen concentration.

In our case, oxygen dosing is used due to the fact that the bell is equipped with an oxygen system and the possibility to control its content. The oxygen dosage in the bell in the case of saturated dives is foreseen for emergency situations, and in deep water dives it is a working procedure. This method of decompression increases the comfort of decompression as it relieves divers from wearing inhaler masks (allowing exhaled oxygen to be ejected outside the chamber) for breathing with the heliox decompression mixture. From the bell, the divers move to a chamber where the atmosphere is filled with a decompression mixture at a selected decompression stop, deeper than $12 \mathrm{~m}$. Once the divers undress to their underwear, at a depth from $12 \mathrm{~m}$ to $3 \mathrm{~m}$, they breathe oxygen in cycles of 25 minutes followed by a 5-minute break from the atmosphere of the chamber. When working at great depths and with relatively long stays, the time spent breathing oxygen from the mask system lasts 4.5 hours, during which the diver's lungs overcome with their muscles the resistance of inhalation and with their elasticity the exhalation resistance. Therefore, low respiratory resistance, especially during exhalation, is very important, as increased resistance turns passive exhalation into effortless active exhalation and can lead to a form of central nervous system oxygen toxicity. extended use of the inhaler mask may cause burns or facial skin infection. To avoid these effects, it is important to maintain the same hygiene and thermal comfort in the chamber.

\section{VARIANT II OF DECOMPRESSION REALISATION}

This method of decompression consists in noninterference with the diving bell atmosphere. The bell is filled with a heliox mixture of a slightly different composition than the working mixture due to the initial filling of the bell with air and the decompression mixture. Divers breathe from the bell atmosphere until they switch to breathing the decompression mixture. The decompression and heliox compression procedures for the diving bell contained in Annex 7 of the Regulation of the Minister of Health of 17 September 2007 provides for conversion of divers to breathing from BIBS inhalers in the diving bell. This reflects the specificity of the French base for deep-sea diving.

At the appropriate time, divers put on bell inhalers (BIBS) supplied with the decompression mixture. During decompression, the oxygen content in the bell's atmosphere increases because the exhalation is directed into the bell's atmosphere. It is obvious that adding oxygen thereby decreases with the depth. For example, for a table depth of $81 \mathrm{~m}$ and a stay of 60 minutes in the stopping zone of 39-30 m, a pair of divers breathing a decompression mixture from inhalers exhale more than $1 \mathrm{~m} 3$ of oxygen into the bell atmosphere breathing per 30 minutes, which increases the oxygen content in the bell to the desired value, corresponding to the decompression mixture.

For work from the beginning of a deep-sea dive zone up to $75 \mathrm{~m}$ and relatively short decompression times using the working mixture, the bell may be filled with the decompression mixture, but only when a pair of divers are diving without the bell operator, and the short decompression using the decompression mixture is performed in the water. This is the optimal method when diving with the bell operator in a 60-75 $\mathrm{m}$ depth zone, when there is no time to replace or enrich the bell atmosphere with oxygen to a value corresponding to the decompression mixture.

Once the oxygen content of the bell reaches $20 \%$, divers can switch to breathing from its atmosphere. Of course, the exhaled carbon dioxide is absorbed by the scrubber, with the ventilator additionally homogenising the mixture in the bell.

We use this method obligatorily when the bell atmosphere is contaminated e.g. with natural gases from a non-tight underwater installation. In his practice, the author encountered one such case when the gas escaped through a leaking valve.

By this method we save helium and oxygen, but we dramatically worsen the comfort during decompression, extending the time of breathing from inhalers.

\section{VARIANT III OF DECOMPRESSION REALISATION}

This is the way in which the divers perform the first phase of decompression in a bell filled with a working mixture. The bell is lifted to the surface and TUP operations are carried out when the working mixture breathing time has elapsed and the divers transition to breathing the decompression mixture, which fills the chamber. This is the easiest way of performing decompression, and the most comfortable for divers, because until the $12 \mathrm{~m}$ stop, when they start to breathe oxygen from the inhalers, they can take off their clothes, use the toilet and rest while lying on the berths and having a relatively long time to breathe the decompression mixture. Previously discussed methods do not offer divers such a long period of rest.

What is stopping us from implementing this method more often? The answer is simple: the cost. For decompression from a $42 \mathrm{~m}$ stop in a hyperbaric chamber, 
we use about $55 \mathrm{~m} 3$ of heliox mixture with $20-22 \%$ oxygen content. If we add to this the consumption of the working heliox with $12-14 \%$ oxygen content by the diver with a 50 minutes stay at $82 \mathrm{~m}$ (about $16 \mathrm{~m} 3$ ) and filling the bell at $78 \mathrm{~m}$ depth $(27 \mathrm{~m} 3)$, a single dive will consume about 98 m3 of heliox mixture. Such a method creates quite a challenge for the supply and financial departments, particularly when the restocking of the mixture is conducted at sea. That is why for the economic use of the heliox decompression mixtures what is important:

- is that the bell immersion is always shallower than the diver's work,

- is the depth (pressure) of the stop at which the divers pass into the chamber,

- $\quad$ is the working mixture used.

In the example above, if the divers go into the chamber at the $21 \mathrm{~m}$ stop, we use almost half of the decompression mixture (about $23 \mathrm{~m} 3$ ), and by immersing the bell at $70 \mathrm{~m}$ we also use $23 \mathrm{~m} 3$ of the working mixture. In total, we will use about $62 \mathrm{~m} 3$ of the heliox mixture. If we use a working heliox mixture with the oxygen content of $18-20 \%$ for diving, we save more than $13 \mathrm{~m} 3$ on the decompression mixture when we perform the TUP operation at a mixture exchange depth of $39 \mathrm{~m}$.

\section{VARIANT IV OF DECOMPRESSION REALISATION}

This method of decompression is very effective from the point of view of helium savings due to the fact that we use air as a decompression mixture. The air to be used during the so-called middle and final phase of decompression and with the heliox/12 $\mathrm{m}$ tables specifying the chamber atmosphere. These tables are for a depth of 50-75 m. They are based on three working mixtures: heliox with $17-18 \%$ oxygen content to a table depth of $78 \mathrm{~m}$, heliox $18-20 \%$ to a table depth of $69 \mathrm{~m}$, and heliox $20-22 \%$ to a table depth of $60 \mathrm{~m}$.

The implementation of decompression with the use of these tables and the use of an open-type bell is similar to the third variant of the decompression realisation except that the heliox decompression mix is replaced by air. The heliox $12 \mathrm{~m} /$ oxygen tables have shorter stay times (in extreme cases by more than 59\%) for the depth zone as compared to the heliox/bell tables. This means that when extending or planning a longer stay of the diver it is necessary to use the heliox tables.

In the case of a $20-22 \%$ heliox, where the working and decompression mixture is the same, the air can be included at any decompression stop specified for the air or decompression mixture.

\section{FINAL REMARKS ON THE IMPLEMENTATION OF DECOMPRESSION IN DEEP- SEA UNDERWATER WORKS IN OUR COUNTRY}

It has been 11 years since the introduction of official tables for deep-sea diving in our country. There is no qualitative analysis of the tables currently used with respect to the domestic diving technique and the quality of its application. These tables have been "adapted" to the technique in our country. During these eleven years, 446 deep dives have been performed in the depth zone of 50$87 \mathrm{~m}$ with stay times of 45-60 min (these are tabular times, in practice they are shorter by 3 to 5 minutes). No case of a decompression incident has been reported. In addition to the positive impact on the decompression of the parameters of the national technique, the organisation and training of divers and crews has an impact on this state of affairs.

In the first stage of implementation of these tables an organisation based on the operation of a pair of divers was used, where both required the same decompression performed in the water in the first stage. During the decompression at the chosen decompression stop they entered the bell while using the working mixture. Using the organisation of saturated dives, this organisation was also introduced in deep water dives as a result of "external influences". With this organisation, the working diver descended into the water outside the bell and the bell operator descended "dry" in the bell (he was not in the water) acting mainly as a backup diver. Such a solution had the advantage of supporting the dive operator in bell operations, during which the working mixture is used, and in performing bell atmosphere stabilisation operations during the transition to breathing with the decompression mixture.

In this type of diving we have two divers with different decompression conditions. For the working diver, the method of decompression is chosen considering the real working depth, and for the bell operator (dressed in a suit with the helmet and equipment removed), who remains in the bell atmosphere at a depth of 5-6 m (and sometimes $12 \mathrm{~m}$ shallower when working on the heads), the method of decompression is chosen in the same way as for the working diver, because it is technically and organisationally impossible to conduct two different decompressions at the same time. For the bell operator, decompression is "very extended". This problem is also not solved by having the latest diving technology, including three-person bell, designed for two divers and an operator. This circumstance has a decisive influence on the diving organisation in the selection of pairs of divers working in a continuous diving cycle every 24 hours from the completion of decompression. The replacement of divers' functions resulting from a fair division of labour also has good effects on decompression, as in every other dive the diver is subject to reduced decompression stress.

The diving technique and open-circuit breathing equipment, as well as the requirements of the heliox/bell decompression tables used, result in a very high helium consumption of the system. Thus, the costs of helium, with all its infrastructure used in the diving system, are very high (especially now that its price has increased by 80 to $100 \%)$. Any possible attempts to reduce helium consumption are not very effective compared to using helium recovery systems and closed-circuit diving equipment. Currently available diving techniques do not allow such helium recovery systems to be installed.

The author sees a chance for improvement in this state of affairs in the following undertakings:

- modernisation of heliox/bell tables aiming at using air from the depth of 12-15 $\mathrm{m}$ in the 50-90 $\mathrm{m}$ zone. Contrary to appearances, this task is feasible for domestic teams,

- parallel formal approval for the use of trimix tables in a $50-90 \mathrm{~m}$ depth zone where air or nitrox is a natural decompression mix,

- where justifiable by the divers' tasks, replace deep-sea diving with saturated diving with 2-3 days' stay even on a relatively shallow saturated plateau, or a multi-level plateau.

The article does not discuss the factors determining the choice of decompression, where its course 
is influenced by such factors that seemingly have nothing to do directly with decompression. These are the diving gratification systems, planning and instrumentation of specific diving works, the non-rhythmicity of underwater works and simplification of logistic processes. However, these are problems that need to be addressed separately.

\title{
REFERENCES
}

US Navy Diving Manual". Published by Direction of Comander of Navy. Revision 7, 2016;

DCIEM Diving Manual part 2 Department of National Defence 1995;

Association of Oil and Gas Procedures "Diving Recommended Practise report No 411 June 2008;

Act of 17 October 2003 on the performance of underwater works;

Regulation of the Minister of Health of 17 September 2007 on health conditions for the performance of underwater works:

Regulation of the Minister of Infrastructure of 19 May 2004 on safety and hygiene at work in the performance of underwater works;

7. S. Skrzyński and the team Targeted Project No. 11/BO contract No. 148 308/C-T00/2001: "Deep dives for the needs of the Marine Rescue". Analysis of foreign deep diving systems in terms of domestic conditions.

\author{
dr inż. Stanisław Skrzyński \\ Akademia Marynarki Wojennej \\ im. Bohaterów Westerplatte \\ 81 - 103 Gdynia 3 \\ ul Śmidowicza 69 \\ tel.: +58626 2746 \\ e-mail: skrzynski@interecho.com
}

